\title{
Familienbezogene Bedarfslagen in Pflegesituationen
}

Katharina Graffmann-Weschke, Marina Otte und Anne Kempchen

\section{Inhaltsverzeichnis}

\subsection{Herausforderung - 104}

7.1.1 Lebensphasen in Familien und die unterschiedlichen Ursachen der Pflegebedürftigkeit - 104

7.1.2 Externe Einflussfaktoren auf familiale Pflegesituationen - 106

7.1.3 Fülle der

Entlastungsmöglichkeiten/Unterstützungsangebote - 107

7.1.4 Wie kann Case Management bei Beratungsbedarfen helfen? - 107

7.2 Die Kunst der Bedarfserfassung - 108

7.2.1 Biographiesensible Pflegeberatung - 109

7.2.2 Pflegeberatung für Kinder und Jugendliche - 111

7.2.3 Methoden der Beratung: ProblemLösen in der Pflegeberatung (PLiP) - 112

7.2.4 Netzwerkarbeit -113

7.3 Zusammenfassung $-\mathbf{1 1 3}$

$$
\text { Literatur - } 115
$$




\section{- Zusammenfassung}

Durch das Case Management als Bestandteil der Pflegeberatung nach \& 7a SGB XI kann auf familienbezogene Bedarfslagen in besonderer Weise eingegangen werden. Die Betrachtung der individuellen Situationen, Herausforderungen und Bedarfe der einzelnen Familienmitglieder in der Pflegesituation erfolgt dabei über die Bewertung der beteiligten Generationen. Die Beeinflussung des Pflegealltags durch die Lebenssituation, die Ursachen der Pflegebedürftigkeit sowie weitere Faktoren werden dazu ebenso betrachtet wie die Auswirkungen der Corona-Pandemie auf die Mitglieder der pflegenden Familie. Beispielhaft werden sensibilisierende Weiterbildungen für die Pflegeberatung vorgestellt und die Bedeutung von Netzwerken für individuelle Bedarfe in der familialen Pflegesituation veranschaulicht.

Case management as a component of care counselling in accordance with $\$ 7$ a Book XI of the Social Code allows family-related needs to be addressed in a special way. The consideration of the individual situations, challenges and needs of the individual family members in the care situation is based on the evaluation of the generations involved. The article considers the influence of the life situation, the causes of the need for care and other factors on everyday care as well as the effects of the corona pandemic on the members of the family providing care. The authors present examples of awareness increasing further training for care counsellors and illustrate the importance of networks to meet the individual needs in the family care situation.

\subsection{Herausforderung}

\subsubsection{Lebensphasen in Familien und die unterschiedlichen Ursachen der Pflegebedürftigkeit}

Bei der Vorstellung eines komplexen Zahnradsystems wird deutlich, wie ein pflegebedürftiges Familienmitglied als einzelnes Rad durch Bewegungen und Veränderungen Einfluss auf die gesamte Familienstruktur ausüben kann. Diese Zusammenhänge, die enorme Komplexität und Bedeutung für alle Beteiligten haben, sollen aus den unterschiedlichen Perspektiven betrachtet werden.

In Familien gibt es die ältere Generation, die mit dem Thema Pflegebedürftigkeit direkt konfrontiert ist - ob als Menschen mit Pflegebedürftigkeit in einem Pflegeheim, als Menschen mit Pflegebedürftigkeit in der ambulanten Versorgung oder als pflegende Angehörige. Die mittlere Generation hat hier die Aufgabe, sich um die ältere und die jüngere Generation zugleich zu kümmern. Ihre Rolle ist vielseitig, denn Zugehörige der mittleren Generation sind selbst Eltern, Kinder, pflegende Angehörige, Ehegatten, berufstätig und vieles mehr. Einige von ihnen erleben über ihre Berufstätigkeit die professionelle Pflege-Rolle. Die jüngste Generation, noch im Kindesalter, ist direkt oder indirekt mit dem Thema Pflege konfrontiert; sie erlebt und begleitet diese täglich mehr oder weniger aktiv. Ggf. sind sie Geschwisterkinder, die zu den 478.915 Kindern und Jugendlichen gehören, die im Alter von 10 bis 19 Jahren als pflegende Angehörige substanzielle pflegerische Aufgaben bei der Pflege und Versorgung in ihrer Familie übernehmen (Metzing 2018, S. 8). Die Mehrheit der Menschen mit Pflegebedürftigkeit ist allerdings älter, wie die aktuelle Auswertung der AOK Nordost veranschaulicht (• Abb. 7.1)

Werden die einzelnen Familienmitglieder in ihren Lebenssituationen betrachtet, wird das Spektrum der Unterstützungsbedarfe ent- 


\section{Analyse der Menschen mit Pflegebedürftigkeit}

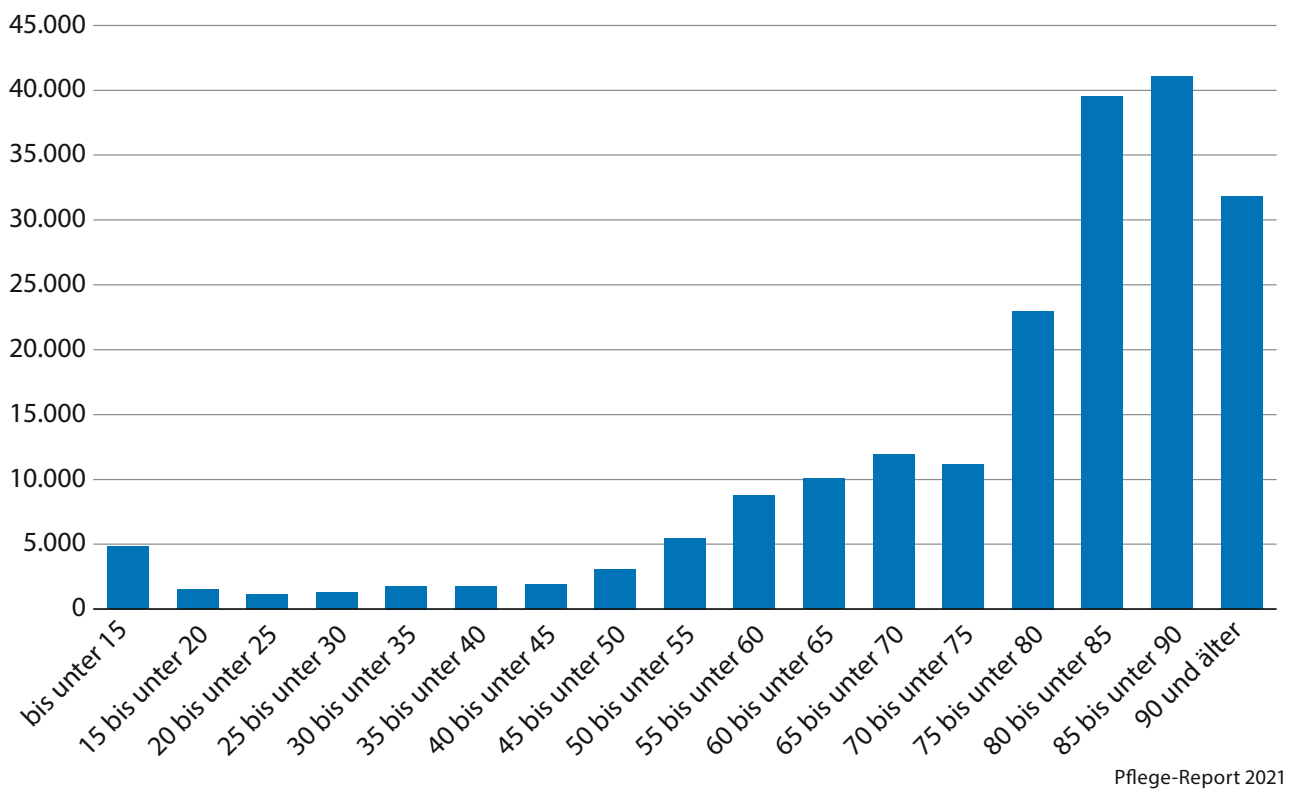

- Abb. 7.1 Analyse der Menschen mit Pflegebedürftigkeit (Pflegekasse bei der AOK Nordost, Stichtag 31.12.2019)

sprechend der Lebenswelt, in der sie sich befinden, sichtbar, z. B. für die Kinder in der Schule, für die Eltern am Arbeitsplatz, für die Großeltern in der eigenen Wohnung.

Bedarfe entstehen auch durch die Ursachen der Pflegebedürftigkeit. $\mathrm{Zu}$ den häufigsten Ursachen zählen chronische Erkrankungen, Akutereignisse mit bleibenden Einschränkungen bei den Verrichtungen des täglichen Lebens, demenzielle Erkrankungen, altersbedingte physische Einschränkungen oder Behinderungen (Hasseler und Görres 2010, S. 17). Die gesundheitlichen Beeinträchtigungen von Kindern sind so vielfältig (z. B. chronisch und schwerkranke Kinder, lebensverkürzend erkrankte oder behinderte Kinder) (Klie und Bruker 2016, S. 7), dass sie zu besonders heterogenen Unterstützungsbedarfen führen.

Andere Bedarfe von Familien entstehen durch weitere Faktoren der individuellen Lebenssituation (z. B. verheiratet, alleinlebend, diverse Lebensentwürfe), die Wohnsituation (z.B. Mehrfamilienhaus in einem Ballungs- gebiet, Wohngemeinschaft, Einfamilienhaus) und Wohnorte (z. B. in einem Dorf oder in einer Stadt, in der Nähe oder weiter entfernt), biografische Hintergründe (z. B. Migrations-, Gewalt- oder auch Kriegserfahrungen), die Unterstützung durch Dritte (z. B. im Mehrgenerationenwohnen) und der Grad der sozialen Einbindung (z. B. in einem Freundeskreis, in einer Kirchengemeinde, durch Ehrenamt, Hobby oder Vereine).

Die konkrete pflegerische Bedarfseinschätzung erfolgt nach Antragstellung bei einer Pflegeversicherung durch den Medizinischen Dienst mit der Prüfung, ob die Voraussetzung einer Pflegebedürftigkeit vorliegt ( $\$ 18$ SGB XI). Dabei steht die Selbstständigkeit in verschiedenen Lebensbereichen im Fokus. Die Ursache für eine Bedarfslage wird im Sinne des neuen Pflegebedürftigkeitsbegriffs ( $\$ 14$ SGB XI) seit 2017 in den Lebensbereichen Mobilität, kognitive und kommunikative Fähigkeiten, Verhaltensweisen und psychische Problemlagen, Selbstversorgung, Bewältigung 
und selbstständiger Umgang mit krankheitsoder therapiebedingten Anforderungen, Belastungen und Gestaltung des Alltagslebens und sozialer Kontakte erfasst (für Erläuterungen hierzu siehe u. a. AOK-Bundesverband 2018). Deren Einschränkungen bestimmen die Festlegung zum aktuellen Bedarf an Unterstützung und die Zuordnung zum entsprechenden Pflegegrad.

\subsubsection{Externe Einflussfaktoren auf familiale Pflegesituationen}

Auch außerfamiliale Faktoren wirken auf Familien, die zu zusätzlichen Belastungen, aber auch Entlastungen führen können. So werden die einzelnen Personen auch durch den medizinischen Fortschritt und die steigende Lebenserwartung beeinflusst. Damit im Zusammenhang stehen die steigende Zahl der Menschen mit Pflegebedürftigkeit und der möglicherweise längere Pflegebedarf; der Fachkräftemangel und regional zunehmend schwierigere Versorgungssituationen. Auch die zahlreichen Einflüsse und Veränderungen durch die Digitalisierung bringen Vorund Nachteile für pflegende Familien mit sich (z. B. Informationsvermittlung, Sicherheit durch technische Assistenz, Videosprechstunden, digitales Lernen, Homeoffice). Digitale Gesundheitskompetenz hat dabei einen entscheidenden Einfluss auf das Finden, Bewerten und Umsetzen von Informationen (vgl. Graffmann-Weschke und Steinmann 2019, S. 28). Die Dynamik der Veränderungen und Möglichkeiten bis hin zu einem „Digitalisierungsschub" (Rothgang und Wolf-Ostermann 2020, S. 37) haben insbesondere durch die CoronaPandemie deutlich zugenommen. Die Kontaktbeschränkungen haben für völlig neue soziale und mediale Zusammenhänge gesorgt, „weil die Lebenswelten der Menschen in ungeahntem Ausmaß auf Medien verwiesen wurden“ (Barberi et al. 2020, S. 1).

Durch die Corona-Pandemie wird die gesamte Gesellschaft in ihrem Alltag existentiell beeinflusst. Für Menschen mit Pflegebedürftigkeit führt sie z. B. auch zu einem erhöhten Mortalitätsrisiko (Rothgang und Wolf-Ostermann 2020, S. 8). Das in der Pflege berufstätige Familienmitglied wird täglich einem erhöhten Infektionsrisiko ausgesetzt. Die Arbeitssituationen in Pflegeeinrichtungen und in der ambulanten Pflege werden beeinflusst aufgrund von ,,zunehmenden Versorgungsaufwänden (u.a. durch Hygieneanforderungen) und gleichzeitigem Personalausfall, der auf eine auch zuvor schon prekäre Personalsituation trifft (...)“ (Rothgang und Wolf-Ostermann 2020, S. 10).

Die Bedeutung von pflegenden Angehörigen wird durch die Corona-Pandemie noch mal viel sichtbarer, denn in ihren Rollen sichern sie weiterhin die Versorgung von Familienangehörigen, ob alt, jung, krank oder pflegebedürftig. In Familien, in denen die Unterstützung der pflegebedürftigen Angehörigen in der eigenen Wohnung durch Arrangements mit Betreuungskräften erfolgte, die nicht mehr nach Deutschland einreisen konnten, mussten gemeinsam mit den Vermittlungsagenturen Lösungen für die Ganztagesbetreuung gefunden werden (vgl. Habel und Tschenker 2020). Menschen mit Pflegebedürftigkeit und ihre Angehörigen standen vor der Wahl, in welchem Umfang die Pflege weiter informell oder formell erbracht werden soll. Schon vor der Corona-Pandemie haben ,ein Viertel der Hauptpflegepersonen insgesamt (...) und fast die Hälfte derjenigen, die zu Beginn der Pflege erwerbstätig waren (...), ihre Erwerbstätigkeit reduziert oder sogar aufgegeben“ (Rothgang und Müller 2019, S. 116). Und die Sorgen um die Familie, die Arbeitsplätze, Isolation und finanzielle Probleme belasten die Familien in der Corona-Pandemie deutlich mehr (vgl. Lorenz-Dant 2020). Teilweise wird die Entscheidung zur Übernahme von mehr Pflegeaufgaben auch zum Schutz der Familie getroffen (Rothgang und WolfOstermann 2020, S. 31). Durch den Verlust der Erwerbstätigkeit und ggf. Kurzarbeit während der Corona-Pandemie gibt es möglicherweise auch mehr Zeit, weiteren Pflegebedarf 
zu erkennen oder auch Pflege zu übernehmen.

\subsubsection{Fülle der Entlastungsmöglichkeiten/ Unterstützungsangebote}

Die vielen möglichen Leistungsansprüche und Beratungsmöglichkeiten, die in mehreren Pflegereformen von 2008 bis $2018^{1}$ eingeführt wurden, sind komplex, sodass die Inanspruchnahme ohne eine hochprofessionelle und zielorientierte Unterstützung fraglich erscheint. Ziel von professioneller Pflegeberatung muss es daher sein, die passenden Leistungen zu finden, zu kombinieren und aus den vielfältigen Versorgungsstrukturen das individuelle Angebot zusammenzustellen. Die meisten pflegenden Familien kennen zwar die zusätzlichen Unterstützungsangebote der gesetzlichen Pflegeversicherung. Die Mehrheit nutzt sie jedoch nicht (Schwinger et al. 2016, S. 199).

Familien in der Pflegesituation benötigen für Entscheidungssituationen immer wieder mehr oder weniger lange und in die Tiefe gehende Informationen und Beratungen. Das ganze Spektrum von einfachen ,Informationen und Auskünften“, über „Beratung“ bis hin zum umfangreichen „Case Management“ ermöglicht der seit 2009 bestehende Rechtsanspruch nach $\S 7$ a SGB XI auf Pflegeberatung für Menschen, die Leistungen der Pflegeversicherung bereits erhalten oder beantragt haben und erkennbar Hilfe, Beratung oder Unterstützung benötigen. Die Pflegeberatung nach $\S 7 \mathrm{a}$ SGB XI wird über die Pflegekassen oder in den Pflegestützpunkten nach $\S 7$ c SGB XI bereitgestellt. Mit dem Pflege-Weiterentwicklungsgesetz (PfWG) von 2008 wurde der Anspruch

1 Gesetz zur strukturellen Weiterentwicklung der Pflegeversicherung (Pflege-Weiterentwicklungsgesetz (PfWG)), Inkrafttreten am 28.05.2008; Erstes Pflegestärkungsgesetz (PSG I), Inkrafttreten am 01.01.2015; Zweites Pflegestärkungsgesetz (PSG II), Inkrafttreten am 01.01.2016; Drittes Pflegestärkungsgesetz (PSG III), Inkrafttreten am 01.01.2017. auf eine umfassende individuelle Pflegeberatung im Sinne eines Fallmanagements eingeführt, das durch das Case Management in der Pflegeberatung (der Qualifizierung und praktischen Umsetzungsmöglichkeit) fest etabliert wurde. Zur Erfüllung dieser Aufgabe wird gemeinsam mit der betroffenen Person und der Pflegeberaterin bzw. dem Pflegeberater ein sogenannter „Versorgungsplan“ erarbeitet, der eine aktuelle Bedarfsermittlung, die Maßnahmenplanung, Zielvereinbarung und Zielerreichung ermöglicht.

\subsubsection{Wie kann Case Management bei Beratungsbedarfen helfen?}

In den Pflegestützpunkten in Berlin wird das Case Management für die sogenannte ,komplexe Beratung“ angewandt. „Die komplexe Beratung richtet sich an Ratsuchende mit umfassenden Problemlagen, mit geringem oder fehlendem Selbsthilfepotential und/oder mehreren Beteiligten. (... ) In der komplexen Beratung findet die Methode des Case Management nach der aktuellen Definition des $\operatorname{DGCC}^{2}(\ldots)$ Anwendung“ (Pflegestützpunkte Berlin 2012). 2019 erhielten 39.000 Ratsuchende „Informationen und Auskünfte", rund 31.000 Ratsuchenden wurde eine „Beratung“ ermöglicht und rund 1.000 Ratsuchende nahmen eine „,komplexe Beratung“ in Anspruch. 2019 fanden dazu 4.496 Hausbesuche statt. Die am häufigsten Kontakt aufnehmenden Personen im Case Management sind mit 42\% Partner und Angehörige (vgl. Träger der Berliner Pflegestützpunkte 2021). Dies wird als Nachweis darauf bewertet, dass der Bedarf an den Beratungen sich nicht allein auf die Menschen mit Pflegebedürftigkeit, sondern ebenso auf deren Familie bezieht. Mit Inkrafttreten der Richtlinie über die einheitliche Durchführung der Pflegeberatung im Jahr 2018 erfolgt die Pflege-

2 Deutsche Gesellschaft für Care und Case Management. 
beratung auf Wunsch der anspruchsberechtigten Person auch gegenüber Angehörigen und weiteren Personen (vgl. GKV-Spitzenverband 2018a), was bis dahin nur in den Pflegestützpunkten vorgesehen war.

Die Bedarfe von Familien zeigen, dass die Herausforderung einer guten und zielführenden Unterstützung eine kontinuierliche Weiterentwicklung und Anpassungen der unterschiedlichen Beratungsansätze in der Pflegeberatung erforderlich machen. Die Pflegesituation in der Corona-Pandemie lässt erkennen, wie schnell Anpassungen an neue Bedarfe, Herausforderungen und Probleme notwendig sind. Mit den sich ändernden multifaktoriellen Rahmenbedingungen in den Wellen der Infektionszahlen durch das Virus SARSCoV-2 muss in der Gesellschaft mit immer wieder neuen aktuellen Situationen umgegangen werden. Familien müssen intern und extern für eine stabile Pflegesituation flexibel und mit relativer Normalität reagieren können.

Während der ersten Welle der Corona-Pandemie ab März 2020 wurde die komplette Pflegeberatung der 74 Pflegestützpunkte in Berlin, Brandenburg und Mecklenburg-Vorpommern auf eine telefonische Beratung umgestellt. Die Pflegestützpunkte in MecklenburgVorpommern erlebten in den ersten drei Quartalen 2020 rund 3.000 telefonische Kontakte mehr als in den ersten drei Quartalen 2019 (Pflegestützpunkte Mecklenburg-Vorpommern 2020). Es wurde sehr deutlich, dass im Notfall auch telefonische Beratungen zur Unterstützung eingefordert werden. Vor allem durch die Nähe und Zusammenarbeit in den Krisenstäben der Träger der Pflegestützpunkte (Pflegekassen, Krankenkassen, Kommunen, Senat in Berlin und Ministerien in Brandenburg und Mecklenburg-Vorpommern) konnten aktuelle gesetzliche Anpassungen zur Entlastung von Familien während der Corona-Pandemie, wie die Flexibilisierung bei Familienpflegezeit und Pflegezeit oder mehr Geld für Pflegehilfsmittel zur Unterstützung der häuslichen Pflege, zeitnah und direkt kommuniziert und in die Familien transportiert werden. Durch die en- ge und stetige Kommunikation zwischen den Mitarbeitenden der Pflegestützpunkte und den Trägern wurden die Nachfragen in den Pflegestützpunkten Seismograf für die Bedarfe und Versorgungslücken und nutzenbringend für die Einschätzung der sozialen Situation in der jeweiligen Region.

\subsection{Die Kunst der Bedarfserfassung}

Die Begriffe der „Pflegeberatung“ und auch des „Case Managements“ sind nicht geschützt. So gibt es in Deutschland kein einheitliches wissenschaftliches Verständnis und auch das Sozialgesetzbuch (SGB) - Elftes Buch (XI) - Soziale Pflegeversicherung als Vorgabe für die „Leistungserbringung“ der Pflegeberatung nutzt den Begriff im Rahmen der Pflege heterogen, nicht nur im $\S 7$ a SGB XI, sondern z. B. auch im Zusammenhang mit dem $\S 37$ „Pflegegeld für selbst beschaffte Pflegehilfen“. Hier ist in Absatz 3 geregelt: „Pflegebedürftige, die Pflegegeld $(\ldots)$ beziehen, haben (..) eine Beratung in der eigenen Häuslichkeit (...) abzurufen. Die Beratung dient der Sicherung der Qualität der häuslichen Pflege und der regelmäßigen Hilfestellung und praktischen pflegefachlichen Unterstützung der häuslich Pflegenden."

Zur Umsetzung der Pflegeberatung für Pflege- und Krankenkassen nach § 7a SGB XI gelten durch die Empfehlungen des GKVSpitzenverbandes nach $\S 7$ a Absatz 3 Satz 3 SGB XI zur erforderlichen Anzahl, Qualifikation und Fortbildung von Pflegeberaterinnen und Pflegeberatern seit 2008 (Empfehlungen des GKV-Spitzenverbandes) aber eindeutige und bindende Qualifizierungsvorgaben von 400 Unterrichtseinheiten (Sozialrecht, Pflege und Case Management), aufbauend auf eine Grundqualifizierung im Bereich der Sozialund Heilpädagogik, Alten-, Gesundheits- und (Kinder-)Krankenpflege oder langjähriger Beratungserfahrung (vgl. GKV-Spitzenverband 2008). 
Seit 2018 gibt es zudem konkrete Hilfestellungen für den gesetzlichen Auftrag durch die Richtlinien des GKV-Spitzenverbandes zur einheitlichen Durchführung der Pflegeberatung nach $\S 7$ a SGB XI (PflegeberatungsRichtlinien). Diese Richtlinien geben einheitliche Maßstäbe und Grundsätze für die Pflegeberatung vor, insbesondere für das Verfahren und die Inhalte der Pflegeberatung als Beratungsprozess (vgl. GKV-Spitzenverband 2018a).

Sowohl durch die Pflegeberatungs-Richtlinien wie auch durch die GKV-Empfehlungen wird berücksichtigt, dass nach $\S 7$ a SGB XI qualifizierte Pflegeberaterinnen und Pflegeberater in ihrer Grundqualifizierung zum Case Management befähigt sind.

In der Evaluation der Pflegeberatung und Pflegeberatungsstrukturen gemäß $\S 7 \mathrm{a}$ Absatz 9 SGB XI von Februar 2020 wird auf die Qualifikationen der Pflegeberaterinnen und Pflegeberater und deren umfassende Weiterbildungsmöglichkeit in den Bereichen Pflegefachwissen, Fallmanagement und Recht eingegangen (Wolff et al. 2020, S. 16). Pflegeberaterinnen und Pflegeberater in den Pflegekassen oder Pflegestützpunkten sind am häufigsten Sozialversicherungsfachangestellte (Wolff et al. 2020, S. 50), denen schwerpunktmäßig Case Management zugeordnet ist (Wolff et al. 2020, S. 66). In der Evaluation werden weitere breite Möglichkeiten der Weiterbildungen für Pflegeberaterinnen und Pflegeberater beschrieben. Diese Vielfalt, ermöglicht durch fehlende starre rechtliche Vorgaben zu Weiterbildungsmöglichkeiten, lässt eine flexible Gestaltung des Qualifikationsrahmens für die Pflegeberatung zu. Damit kann auf Bedarfe und Bedürfnisse der Familien individuell und regionsspezifisch eingegangen werden. Die ausführenden Pflegeberaterinnen und Pflegeberater können als Lotse des Systems bezeichnet werden, der hilft, die passende zielführende Unterstützung $\mathrm{zu}$ finden und deren Zugang zu erlangen.

\subsubsection{Biographiesensible Pflegeberatung}

Durch die Aktualisierung der Empfehlungen des GKV-Spitzenverbandes 2018 haben Pflegeberaterinnen und Pflegeberater (vgl. GKVSpitzenverband 2018a) regelmäßig an themenspezifischen Weiterbildungen, die der Kompetenzerweiterung dienen, teilzunehmen. Um den vielen besonderen Unterstützungsbedarfen in der Beratung Rechnung zu tragen, werden deshalb zunehmend Weiterbildungen $\mathrm{zu}$ speziellen Themen bereitgestellt (z.B. zur Kultursensiblen Pflegeberatung/Vielfalt, Suchtsensiblen Pflegeberatung, zur Zielgruppe der Familien mit pflegebedürftigen Kindern) und Methoden zur Beratung weiterentwickelt (z. B. ProblemLösen in der Pflegeberatung (PLiP)), die an dieser Stelle beispielhaft und in ihrem bedarfsorientierten Aufbau vorgestellt werden sollen.

Allen hier beschriebenen Weiterbildungen ist gemeinsam, dass jede einzelne zur weiteren Professionalisierung der Pflegeberaterinnen und Pflegeberater beiträgt. Sie sollen nicht zur Übernahme noch weiterer Beratungsaufgaben als zusätzliche Aufgaben beitragen, sondern durch Sensibilisierung den Blick und das Verständnis auf ausgewählte Bedarfe ausweiten, die Ansprache zu besonderen Themen individuell ausgerichtet professionalisieren und dann, wenn tiefer gehende Beratung notwendig ist, zu weiteren speziellen Beratungsstellen (z. B. der Suchtberatung) lotsen, um ggf. gemeinsam tätig zu werden.

\section{- Kultursensible Pflegeberatung und Vielfalt}

Weiterbildungen zur Kultursensiblen Pflegeberatung mit Zusatzmodulen zu Menschen mit Demenz und bei Menschen am Lebensende sowie zur Vielfalt in der Pflege ermöglichen die Auseinandersetzung der Pflegeberaterinnen und Pflegeberater mit den unterschiedlichsten notwendigen Perspektiven. Für sie bedeutet die Beschäftigung mit Kultursensibilität, ,sich mit der eigenen Kultur und Kul- 
turidentität auseinanderzusetzen, eigene Werte und Normen zu reflektieren und zu lernen, persönliche Sichtweisen und Annahmen differenziert zu hinterfragen. Eine kultursensible Pflegeberatung ist eine biographiesensible Beratung, wobei alle Biographien individuell verschieden sind. Ziel ist es, den vielfältigen Lebensentwürfen, denen man in der Beratung begegnet, gerecht zu werden. Personen mit einem Migrationshintergrund sind keine homogene Gruppe, es ist daher weder möglich noch nötig, eine Expertise jeder Kultur zu erwerben." (Schmidt-Pabst und GraffmannWeschke 2019, S. 1)

Für Familien mit Migrationserfahrung ist neben der Hürde in der Verständigung in unterschiedlichen Sprachen das gegenseitige kulturelle Verständnis für eine Pflegesituation unerlässlich. Als Hürde vor den Zugängen zu Hilfesystemen steht der Zugang zur Pflegeberatung. Das Projekt der Interkulturellen BrückenbauerInnen in der Pflege (IBIP) ermöglicht hier seit Jahren in enger Zusammenarbeit mit den Pflegestützpunkten in Berlin niedrigschwellige Ansprache und Begleitung (vgl. GKV-Spitzenverband 2018b). Sprach- und Kulturmittlerinnen und Kulturmittler begleiten dabei Pflegeberaterinnen und Pflegeberater der Pflegestützpunkte wie auch den Medizinischen Dienst bei der Pflegebegutachtung und vermitteln durch ihre Kenntnisse zwischen Beratungsstrukturen, Pflegeeinrichtungen und Menschen mit Pflegebedürftigkeit und Migrationshintergrund und deren Angehörigen. Auch beim entsprechenden Case Management spielt es eine groBe Rolle, Angebote und Dienstleister sprachund kultursensibel zu vermitteln, wie das folgende Beispiel zeigen soll: Eine Tochter wandte sich mit der Bitte um Hilfe für ihre pflegebedürftige Mutter an eine Brückenbauerin, die den Kontakt zu dem Pflegestützpunkt im Bezirk herstellte. Die Mutter selbst, erkrankt mit einer lebensverkürzenden onkologischen Diagnose, lehnt bisher professionelle Hilfe ab. Die Mutter ist als junge Frau aus der Türkei nach Deutschland gezogen. Sie hat ihr Leben stets auf ihre vier Kinder ausgerichtet. Ihr kulturell geprägtes Bild von Familie bedeutet, dass sie die Versorgung ausschließlich durch die Familie erwartet. Die häusliche Versorgung und Begleitung allein durch die Familie ist aber nur begrenzt möglich. Durch die Brückenbauerin wurde ein kultursensibles Case Management möglich, sodass bei der Planung von Maßnahmen und Zielen die biographiespezifischen Bedarfe berücksichtigt werden konnten. Durch die Vernetzung der Pflegeberaterin des Pflegestützpunktes und der Brückenbauerin konnten Dienstleister entsprechend ihrer Sprachkompetenz ausgewählt werden, mit denen die Mutter einverstanden ist. Ein palliativ behandelnder Onkologe, ein türkischer Pflegedienst und ein interkultureller Hospiz- und Palliativdienst versorgen die Mutter und gehen kultur- und sprachsensibel auf ihre Wünsche und Bedarfe ein. In Situationen, in denen es für deutsche Fachbegriffe keine passende wörtliche Übersetzung gibt, lassen sich in der Muttersprache Zusammenhänge erklären und so können Gespräche und die Versorgung bedarfsgerecht und in Übereinstimmung aller Beteiligten ermöglicht werden.

Biographiesensible Beratungs- und Versorgungsbedarfe für die Pflegesituation der älter werdenden Generation entstehen z.B. durch Migration, aber auch durch die Vielfalt von Lebensentwürfen, wie sie vor allem in Großstädten wie Berlin häufiger gelebt werden. So ergeben sich in den Pflegestützpunkten auch Bedarfe, die sich z. B. auf spezifische Fragen zu Sexualität und Geschlecht im Alter und bei Pflegebedürftigkeit beziehen, wie beispielsweise nach weltoffenen, toleranten Pflegeeinrichtungen oder Sexualtherapeuten. Eine entsprechende Weiterbildung zum Thema Vielfalt und LSBTI $^{3}$ wurde deshalb durch die Senatsverwaltung für Gesundheit, Pflege und Gleichstellung der Stadt Berlin, die Fachstelle für pflegende Angehörige, dem Sprecher*innenrat der Berliner Pflegestützpunkte, die Schwulenberatung Berlin und RuT - Rat

3 „LSBTI* ... ist die Abkürzung für Lesben, Schwule, Bisexuelle, Trans* und Inter*. Lesben, Schwule und Bisexuelle fallen in das Spektrum sexueller Vielfalt. Trans* und Inter* in das Spektrum geschlechtliche Vielfalt.“(Schwulenberatung Berlin 2020, S. 6). 
und Tat: Offene Initiative Lesbischer Frauen e. V. in Berlin-Neukölln für alle Mitarbeitenden der Berliner Pflegestützpunkte entwickelt und durchgeführt.

\section{- Suchtsensible Pflegeberatung}

Die Weiterbildung zur Suchtsensiblen Pflegeberatung war 2017 eins der ersten deutschlandweiten Angebote speziell für Pflegeberaterinnen und Pflegeberater der Kranken-/Pflegekassen und Pflegestützpunkte, welche die Fachstelle für Suchtprävention Berlin gGmbH durch eine Multiplikatorenschulung für Fachstellen der Suchtprävention aller Bundesländer und der Schweiz ermöglicht hat. Wissenschaftliche Erkenntnisse zur Bedeutung von Sucht im Alter bestärkten dabei die Bedarfe aus der Beratung, auch schambesetzte Tabuthemen professioneller ansprechen zu können und einer ,stärkeren Orientierung an individuellen Beratungsinhalten“ (Jüngling et al. 2020, S. 3) für die Pflegeberatung den Weg zu bereiten.

Aufbauend auf die 400-stündige Qualifizierung zur Pflegeberatung nach § 7a SGB XI ist das Thema Sucht ein besonderes Beispiel dafür, welche Facetten sich in Weiterbildungen für Pflegeberaterinnen und Pflegeberater ergeben. In einer Arbeitssituation einer Mitarbeiterin oder eines Mitarbeiters z. B. in einem Pflegestützpunkt kann das Thema beispielsweise einen Menschen mit Pflegebedürftigkeit betreffen (z. B. durch die Einnahme von regelmäßig 20 Medikamenten täglich), Familienangehörige in einer Überlastungssituation (z. B. zu Schlafmitteln bei Schlafstörungen), professionelle Pflegefachpersonen des ambulanten Pflegedienstes oder in einer Pflegeeinrichtung (z. B. zum Umgang mit der Bereitstellung von Alkohol, das Bier oder der Wein am Abend), im eigenen Familienumfeld (z.B. der drogenabhängige Neffe) und im Arbeitskontext (z.B. die Kollegin mit regelmäßigem Alkoholgeruch). Um daraus einen Handlungsbedarf $\mathrm{zu}$ erkennen, eine professionelle Ansprache zu ermöglichen und Lösungen herbeizuführen, hat die Weiterbildung den Anspruch, auch die eigene Einstellung zum Konsum von z. B. Alkohol oder Medikamenten zu überdenken.
Zudem ist Wissen im Kontext von Sucht im Alter und in der Pflege zu erlangen, zu dem beispielsweise gehört, dass der Alkoholkonsum von Menschen im Alter sinkt (Weyer et al. 2006) und Polypharmazie im Alter zunimmt (ABDA 2020). Aufbauend auf die Erkenntnisse von Abhängigkeitsentwicklungen im Alter werden die Chancen der motivierenden Kurzintervention für die Pflegeberatung geübt, um Lösungen für die Betroffenen ggf. gemeinsam mit den entsprechenden Suchtberatungsstellen in den Bundesländern anzubahnen.

\subsubsection{Pflegeberatung für Kinder und Jugendliche}

Es gibt Kinder mit eigenem anerkannten Pflegebedarf und Geschwister, die in ihrer Familie eine Pflegesituation, z. B. von Bruder oder Schwester oder Großeltern, begleiten. Eine Pflegeberatung muss dann auch Kompetenzen zu den besonders heterogenen Beratungsbedarfen von Familien mit pflegebedürftigen Kindern haben und sensibilisiert sein für Kinder und Jugendliche mit Pflegebeteiligung.

Die Unterschiede in der Beratung bei Themen der jüngeren Generation gegenüber den Maßnahmen zu erwachsenen Menschen mit Pflegebedürftigkeit beziehen sich dabei u.a. auf die Lebenssituation, die Auswirkungen auf andere Familienmitglieder und die Anforderungen an ein Netzwerk. Bei pflegebedürftigen Erwachsenen beinhalten die Gespräche der Pflegeberatung die Sorge, Begleitung und Unterstützung für eine Lebensphase, die viele Jahre dauern kann, in der Regel im Bedarf fortschreitet und mit dem Tod endet. In dieser Zeit geht es darum, Lebensqualität in der letzten Lebensphase zu ermöglichen. Bei Familien mit hilfe- und pflegebedürftigen Kindern besteht Beratungsbedarf aufgrund der Notwendigkeit, viele weitere Akteure einzubeziehen, um eine weiterreichende Versorgung mit einer gewissen Normalität für alle Familienangehörigen $\mathrm{zu}$ ermöglichen, z. B. auch durch den Besuch eines Kindergartens und von Schulen. 
Familien mit pflegebedürftigen Kindern sind finanziell, körperlich und psychisch stark belastet. Wenn die Ursachen des Pflegebedarfs in einer Erkrankung liegen, werden lediglich $50 \%$ der Eltern zeitnah über die Erkrankung ihres Kindes und die Konsequenzen informiert. Unterstützung in Hinblick auf die psychischen Belastungen erhalten nur ein Viertel aller betroffenen Familien und auch auf allgemeine Hilfen und Hilfsangebote wird häufig nicht hingewiesen. Für diese Situation wünschen sich die Familien einen verlässlichen und kundigen Lotsen und eine einzige Anlaufstelle für ihre Beratungsbedarfe. Durch den hohen Betreuungsbedarf nehmen die Eltern auch berufliche Einschränkungen in Kauf, die zu finanziellen Einbußen führen können. (vgl. Kofahl und Lüdecke 2014, S. 10 ff.)

In der Situation der Pflege von Kindern hat die Organisation von informellen Informationen durch Zusammenschlüsse im Rahmen von Selbsthilfegruppen eine besondere Bedeutung. Damit wird zum Teil aufgefangen, dass die Familien sich durch ein fehlendes Schnittstellenmanagement in ihrer Situation allein gelassen fühlen (vgl. Berliner Senatsverwaltung für Gesundheit und Soziales 2015, S. 27 ff.).

Die Berücksichtigung der besonderen Bedarfe der Familien, in denen Kinder und Jugendliche aus den unterschiedlichsten Gründen gepflegt werden, erfolgt in einer einwöchigen Qualifizierung für Pflegeberaterinnen und Pflegeberater. Sie wurde aufbauend auf dem Niveau des Case Managements der Deutschen Gesellschaft für Case und Care Management (DGCC) entwickelt und berücksichtigt Erfahrungen zu den ,frühen Hilfen“, den „,Kinderbeauftragten“ der Berliner Pflegestützpunkte und der Selbsthilfe. Auch die Bedarfe von ,jungen Pflegenden“, der Kinder und Jugendlichen, die beispielsweise als Geschwisterkinder in die Pflegesituation mit einbezogen sind, werden darin berücksichtigt. In Ergänzung zur Sensibilisierung auf Unterstützungs- und Beratungsbedarfe werden dabei Verantwortlichkeiten in Netzwerken aufgezeigt und zum Aufbau eines tragfähigen Netzes ermutigt, das die Themenkomplexe der Bildung (Kita, Schule),
Jugendhilfe und psychologischen Betreuung beinhaltet. Ein besonderer Fokus liegt auch auf der Partizipation der pflegebedürftigen Kinder und Jugendlichen und auf dem Übergang in die Erwachsenenversorgung, die Transition, wozu weitere Akteure in die Vernetzung einbezogen werden (vgl. Zoller und Graffmann-Weschke 2020).

\subsubsection{Methoden der Beratung: ProblemLösen in der Pflegeberatung (PLiP)}

Die Methoden, die im Rahmen des Case Managements der Pflegeberatung angewendet werden, entwickeln sich ständig weiter. Gerade in Bezug auf Familien und die Tatsache, dass im Rahmen des Case Managements in der Regel unterschiedliche Angehörige eingebunden sind, soll als Beispiel das Assessment ProblemLösen in der Pflegeberatung vorgestellt werden. Dieses wurde im Rahmen zweier vom GKV-Spitzenverband nach $\S 8$ Abs. 3 SGB XI geförderter Modellvorhaben zur Weiterentwicklung der Pflegeversicherung sowie weiterer vom Bundesministerium für Bildung und Forschung geförderter Studien entwickelt (GKV Spitzenverband 2018c). PLiP unterstützt pflegende Angehörige dabei, mit den Herausforderungen bei der Pflege des pflegebedürftigen Angehörigen umzugehen. Auch die Pflegeberatungs-Richtlinie erwähnt diese Assessmentmethode bei der Beratung von pflegenden Angehörigen.

Bei dieser Methode stehen die pflegenden Angehörigen in Bezug auf die Bedarfe des Menschen mit Pflegebedürftigkeit im Mittelpunkt. Sie betrachten mit den Pflegeberaterinnen und Pflegeberatern u. a. anhand eines Kartensets ihre Probleme und Ressourcen und entscheiden, ob und was sie in der aktuellen Pflegesituation ändern wollen. Mit dieser Methode werden die Angehörigen besonders dabei unterstützt, eigene Lösungsmöglichkeiten zu entwickeln. Dadurch können insbesondere belastende Situationen für die Angehöri- 
gen identifiziert, minimiert oder sogar behoben werden. In den dabei geführten Gesprächen kommen vor allem auch präventive, die körperliche oder psychische Gesundheit stärkende Maßnahmen zur Anwendung. Zur Durchführung dieser Methode ist eine entsprechende Weiterbildung notwendig, in der ein besonderes Augenmerk auf die Pflegeberaterinnen und Pflegeberater selbst einschließlich deren Supervision gelegt wird (vgl. Pfeiffer et al. 2017).

\subsubsection{Netzwerkarbeit}

Die Voraussetzung für ein Case Management in der Pflegeberatung beruht auf der Kenntnis und dem Kontakt zu regionalen Angeboten der Prävention, Unterstützung und Entlastung für die Pflege und darüber hinaus. Insbesondere bei den Pflegestützpunkten, die als regionale neutrale Beratungsstellen auch den gesetzlichen Auftrag zur Netzwerkarbeit nach $\S 7 c$ SGB XI haben, wird die Bedeutung einer Zusammenarbeit aller Beteiligten vor Ort sichtbar. Die Mitarbeiterinnen und Mitarbeiter können dadurch einen passenden Versorgungs-Mix anbieten, der z. B. auch über kostenpflichtige Leistungen der Pflegeversicherung hinausgeht. Beispielsweise können so eine Tagespflege für Menschen mit Demenz, eine Nachbarschaftshilfe für das Einkaufen, die Spaziergangsgruppe der Kirchengemeinde und die Selbsthilfegruppe für pflegende Angehörige des Landkreises kombiniert werden.

Der in einer Pflegeberatung erstellte Hilfebzw. Versorgungsplan legt vereinbarte Maßnahmen und Ziele fest. Die Ziele werden darauf aufbauend durch die Zusammenarbeit im Netzwerk erreicht. Jeder individuelle Fall erfordert unterschiedliche und vielfältige Netzwerkpartner. Zusätzlich zu den gängigen Partnern wie Ärztinnen und Ärzten, Krankenhäusern, Apotheken, ambulanten Pflegediensten, stationären Pflegeheimen, Anbietern in der Essensversorgung, Tagespflegen, spezialisier- ten Beratungspartnern (z.B. die Alzheimer Gesellschaften) muss in manchen Fällen das Netzwerk erweitert werden.

Für die Unterstützung von Familien mit pflegebedürftigen Kindern und Jugendlichen könnten z. B. spezialisierte Kinderpflegedienste, regionale Jugendämter, der Sozialpädiatrische Dienst sowie die Zusammenarbeit mit Schulberatern und -psychologen notwendig sein.

Bei Familien mit Gewaltproblematiken in der Pflegesituation ist die Einbindung und $\mathrm{Zu}$ sammenarbeit mit spezialisierter Beratung wie z. B. den Beratungsstellen „Pflege in Not“, die in einzelnen Bundesländern vertreten sind, für alle Beteiligten, die Betroffenen wie auch die Beraterinnen und Berater hilfreich.

Bei dieser Aufzählung wird bereits deutlich, dass Netzwerkarbeit auf die Region, in welcher der Fall auftritt, fokussiert. Dazu nutzen Pflegeberaterinnen und Pflegeberater im Alltag z.B. Netzwerkkarten, die individuell angepasst werden (- Abb. 7.2).

In der Zusammenarbeit der Partner ist die Intensität fallabhängig. In den meisten Situationen bleibt es bei einer einfachen Kommunikation, z. B. der Vermittlung zu einer Sprechstunde der Alzheimer Gesellschaft. Andere Situationen erfordern eine aktivere Moderation der Situation. Dabei können sich auch regelmäßige Fallkonferenzen zwischen allen Beteiligten entwickeln, in denen die Situation gemeinsam beraten wird.

\subsection{Zusammenfassung}

Durch das Case Management als Bestandteil der Pflegeberatung nach § 7a SGB XI kann auf familienbezogene Bedarfslagen in besonderer Weise eingegangen werden. Die Betrachtung der Situationen, Herausforderungen und Bedarfe der einzelnen Familienmitglieder in der Pflegesituation erfolgt dabei über die Bedarfe der unterschiedlichen beteiligten Generationen. Die Beeinflussung des Pflegealltags durch die Lebenssituation, die Ursachen der Pflege- 


\begin{tabular}{|l|l|}
\hline Beispiel: Netzwerkkarte Pflegestützpunkt & Behandler \\
\hline Pflege/Versorger & Ärztinnen und Ärzte/ SAPV-/ \\
\hline Pflegende Angehörige, Nachbarn, Freunde & Palliativärztinnen und Palliativärzte \\
Niedrigschwellige Betreuungsangebote & Psychologinnen und \\
Tagespflegeeinrichtungen & Psychologen/Psychotherapeutinnen und \\
Spezialisierter ambulante & Psychotherapeuten \\
Palliativpflegedienst (SAPV Pflegedienst) & Krankenhaus-Sozialdiente \\
Nachbarschaftshilfe & Gedächtnissprechstunde \\
Kurzzeitpflege/Stationäre & Psychiatrische Institutsambulanzen \\
Pflegeeinrichtungen & Therapeuten-Physiotherapeuten, \\
Fahrbarer Mittagstisch & Ergotherapeuten/Logopäden \\
Sanitätshäuser & Medizinische Fußpflege \\
Haushaltsnahe Dienstleister & \\
Mobilitätshilfedienste & \\
\hline Kostenträger/Entscheider & Beratung/Kontakt \\
\hline Krankenversicherung/Pflegeversicherung & Betreuungsverein-Beratung/Weiterbildung \\
Bezirksamt/Landkreis & ehrenamtlicher Betreuer \\
Gesundheitsamt & Spezialisierte Beratungsstellen-Demenz \\
Jugendamt & Pflege in Not \\
Amtsgericht/Betreuungsbehörde & Krisendienst \\
& Sozialpsychiatrischer Dienst \\
& Selbsthilfe \\
\hline & Kieztreff/Nachbarschaftshaus \\
\hline
\end{tabular}

- Abb. 7.2 Netzwerkkarte für den Pflegestützpunkt (Pflegeberatung der AOK Nordost)

bedürftigkeit sowie weiterer Faktoren werden dazu ebenso einbezogen wie die Auswirkungen der Corona-Pandemie auf die Mitglieder der pflegenden Familie.

Um die vielfältigen gesetzlichen Entlastungs- und Unterstützungsmöglichkeiten wie auch regionale Angebote, z. B. aus der Selbsthilfe, der Familie adäquat und passgenau näherzubringen, erhalten die Pflegeberaterinnen und Pflegegeberater der Krankenkassen, Pflegekassen und Pflegestützpunkte eine 400-stündige Qualifizierung und nach den Vorgaben der Pflegeberatungs-Richtlinie seit 2018 auch bedarfsgerechte Weiterbildungen.

Diese tragen dazu bei, dass das multiprofessionelle Talent - Pflegeberatung mit Case
Management - zunehmend sensibilisiert, geschärft in den eigenen Einstellungen und professioneller wird. Anhand von Beispielen werden die Kompetenzentwicklung der Beraterinnen und Berater für biographiesensible Beratungen, die besonderen Anforderungen für die Beratung $\mathrm{zu}$ pflegebedürftigen Kindern und die Beratungsmethode ProblemLösen in der Pflegeberatung (PLiP) erläutert. Den jeweiligen Fall mit seiner Bedarfslage sowie seinen unterschiedlichen Herausforderungen zu analysieren, die richtigen Ziele und Maßnahmen zu definieren und mit Hilfe von Netzwerken zu begleiten erfordert Qualifikation, Erfahrung und Engagement. Die Kunst der Bedarfserfassung will gelernt und geübt sein. 


\section{Literatur}

ABDA (Arbeitsgemeinschaft der Berufsvertretungen Deutscher Apotheker) (Hrsg) (2020) ABDA-Faktenblatt-Polymedikation

AOK-Bundesverband (2018) Pflege neu verhandeln. Das umfassendere Verständnis von Pflegebedürftigkeit im Pflegealltag leben. AOK-Bundesverband, Berlin

Barberi A, Grünberger N, Schmölz A (2020) Editorial 2/2020 Nähe(n) und Distanz(en) in Zeiten der COVID-19-Krise. Medienimpulse 58(2):1-20. https:// doi.org/10.21243/mi-02-20-32

Berliner Senatsverwaltung für Gesundheit und Soziales (2015) Maßnahmenplan pflegende Angehörige. Weiterentwicklung des Berliner Unterstützungssystems. Berliner Senatsverwaltung für Gesundheit und Soziales, Berlin

GKV-Spitzenverband (Hrsg) (2008in) Empfehlungen des GKV-Spitzenverbandes nach $\S 7$ a Absatz 3 Satz 3 SGB XI zur Anzahl, Qualifikation und Fortbildung von Pflegeberaterinnen und Pflegeberatern vom 29. August 2008 in der jeweiligen aktualisierten Fassung

GKV-Spitzenverband (Hrsg) (2018a) Richtlinien des GKV-Spitzenverbandes zur einheitlichen Durchführung der Pflegeberatung nach § 7a SGB XI vom 07. Mai 2018 (Pflegeberatungs-Richtlinien)

GKV-Spitzenverband (2018b) Modellvorhaben zur Weiterentwicklung der Pflegeversicherung nach $\S 8$ Abs. 3 SGB XI. Abgeschlossene Modellvorhaben. IBIP - Interkulturelle BrückenbauerInnen in der Pflege

Spitzenverband GKV (2018c) Problemlösen in der Pflegeberatung - ein Ansatz zur Stärkung der Pflegeberatung nach $\S 7$ a SGB XI. Schriftenreihe Modellprogramm zur Weiterentwicklung der Pflegeversicherung, Bd. 14

Graffmann-Weschke K, Steinmann (2019) Digitale Gesundheitskompetenz: Eine Bedarfsermittlung bei Versicherten der AOK Nordost. In: Universität Bielefeld, Fakultät für Gesundheitswissenschaften, Interdisziplinäres Zentrum für Gesundheitskompetenzforschung (Hrsg) 2. Internationales Symposium, Gesundheitskompetenz - Forschung, Praxis, Politik, 2./3. Mai 2019, Abstractband. Universität Bielefeld, Fakultät für Gesundheitswissenschaften \& Fakultät für Erziehungswissenschaft, Interdisziplinäres Zentrum für Gesundheitskompetenzforschung, Bielefeld, S 28-29

Habel S, Tschenker T (2020) Stay At Work. Zur Situation der Live-In-Pflege in der Corona-Krise. Soziale Sicherh 6:215-219

Hasseler M, Görres S (2010) Was Pflegebedürftige wirklich brauchen ... Zukünftige Herausforderungen an eine bedarfsgerechte ambulante und stationäre pflegerische Versorgung. Schlütersche, Hannover

Jüngling K, Schmidt A, Graffmann-Weschke K (2020) Suchtsensible Pflegeberatung. Suchtgefährdung er- kennen und professionell intervenieren, 2. Aufl. Fachstelle für Suchtprävention Berlin gGmbH, Berlin

Klie T, Bruker C (2016) Versorgungskoordination bei Familien mit schwer und lebensverkürzend erkrankten Kindern - Expertise. Institut für angewandte Sozialforschung an der Ev. Hochschule Freiburg, Freiburg

Kofahl C, Lüdecke D (2014) Familie im Fokus Die Lebens- und Versorgungssituation von Familien mit chronisch kranken und behinderten Kindern in Deutschland. Ergebnisse der Kindernetzwerk-Studie. AOK Bundesverband, Berlin

Lorenz-Dant K (2020) International examples of measures to support unpaid carers during the COVID-19 pandemic. International Long Term Care Policy Network (Itcovid.org)

Metzing S (2018) Abschlussbericht zum Projekt „Die Situation von Kindern und Jugendlichen als pflegende Angehörige“. Universität Witten/Herdecke, Department für Pflegewissenschaft, Witten

Pfeiffer K, Hautzinger M, Patak M et al (2017) Problemsolving in caregiver-counselling (PLiP Study): study protocol of a cluster randomized pragmatic trial. BMC Geriatr 17(64)

Pflegestützpunkte Berlin - AG Qualität der Pflegestützpunkte Berlin (2012) Modul komplexe Beratung zu den Qualitätsstandards. Beratung nach § 7c SGB XI einschließlich soziale Beratung und Pflegeberatung $\S 7$ a SGB XI in den Pflegestützpunkten Berlin. Unveröffentlichte Quelle

Pflegestützpunkte Mecklenburg-Vorpommern (2020) Pflegeberatung zum Stichtag 1. Okt. 2020 in den Pflegestützpunkten Mecklenburg-Vorpommern. Unveröffentlichte Quelle

Rothgang H, Müller R (2019) Barmer-Pflegereport 2019. Ambulantisierung der Pflege. zweiband.media, Berlin

Rothgang H, Wolf-Ostermann K (2020) Zur Situation der häuslichen Pflege in Deutschland während der Corona-Pandemie. Ergebnisse einer Online-Befragung von informellen Pflegepersonen im erwerbstätigen Alter

Schmidt-Pabst E, Graffmann-Weschke K (2019) Kultursensibel Pflegeberatung für Sozial- und Pflegeberaterinnen und -berater der Kranken-/Pflegekassen und Pflegestützpunkte. Berlin. Unveröffentlichte Quelle

Schwinger A, Tsiasioti C, Klauber J (2016) Unterstützungsbedarf in der informellen Pflege - eine Befragung pflegender Angehöriger. In: Jacobs K, Kuhlmey A, Greß S, Klauber J, Schwinger A (Hrsg) Pflege Report 2016. Schattauer, Stuttgart, S 189-216

Schwulenberatung Berlin gGmbH (2020) Weil ich so bin, wie ich bin. Vielfalt in der Pflege. Ein Praxis-Leitfaden für stationäre und ambulante Dienste. Schwulenberatung Berlin $\mathrm{gGmbH}$, Berlin

Träger der Berliner Pflegestützpunkte (2021) Statistik der Berliner Pflegestützpunkte für das Jahr 2019. Unveröffentlichte Quelle 
Weyer S, Schäufele M, Hendlmeier I (2006) Alkoholmissbrauch und -abhängigkeit bei Bewohnern und Bewohnerinnen in Altenpflegeheimen. Repräsentative Ergebnisse aus der Stadt Mannheim. Z Gerontopsychologie -psychiatrie 19(4):229-235

Wolff JK, Pflug C, Rellecke J, Rieckhoff S, Dehl T, Nolting H-D (2020) Evaluation der Pflegeberatung und Pflegeberatungsstrukturen gemäß $\S 7$ a Absatz 9 SGB XI. IGES Institut im Auftrag des GKV-Spitzenverbandes, Berlin
Zoller E, Graffmann-Weschke K (2020) Curriculum Pflegeberatung nach $\S 7$ a SGB XI mit dem Schwerpunkt Kinder und Jugendliche für Pflegeberaterinnen und -berater der Kranken-/Pflegekassen und Pflegestützpunkte. Berlin. https:// pflegeakademie.aok.de/uploads/news/documents/ Curriculum-Pflegeberatung-Kinder.pdf. (Zugegriffen: 22. März 2021)

Open Access Dieses Kapitel wird unter der Creative Commons Namensnennung 4.0 International Lizenz (http:// creativecommons.org/licenses/by/4.0/deed.de) veröffentlicht, welche die Nutzung, Vervielfältigung, Bearbeitung, Verbreitung und Wiedergabe in jeglichem Medium und Format erlaubt, sofern Sie den/die ursprünglichen Autor(en) und die Quelle ordnungsgemäß nennen, einen Link zur Creative Commons Lizenz beifügen und angeben, ob Änderungen vorgenommen wurden.

Die in diesem Kapitel enthaltenen Bilder und sonstiges Drittmaterial unterliegen ebenfalls der genannten Creative Commons Lizenz, sofern sich aus der Abbildungslegende nichts anderes ergibt. Sofern das betreffende Material nicht unter der genannten Creative Commons Lizenz steht und die betreffende Handlung nicht nach gesetzlichen Vorschriften erlaubt ist, ist für die oben aufgeführten Weiterverwendungen des Materials die Einwilligung des jeweiligen Rechteinhabers einzuholen.

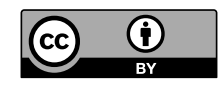

